\title{
Starvation ketoacidosis in a patient with gastric banding
}

\author{
A Lulsegged, E Saeed, E Langford, C Duffield, S El-Hasani and N Pareek
}

\section{Case presentation}

A 31-year-old lady presented to hospital with a two-week history of shortness of breath, pleuritic chest pain and productive cough. She had been unable to keep solids down for the previous month and was only able to tolerate small sips of fluid before vomiting for the week prior to admission. She had a gastric band fitted one year previously and had lost $50 \mathrm{~kg}$ since, but had no history of diabetes mellitus. She had no previous psychiatric history and denied taking excess alcohol, anti-freeze or tricyclic antidepressants.

On examination, blood pressure was $128 / 93 \mathrm{mmHg}$, pulse $108 /$ minute, respiratory rate $18 /$ minute and oxygen saturation $96 \%$ on room air. She looked pale, chest and abdominal examination were unremarkable. Initial arterial blood analysis demonstrated a normal pH (7.372), $\mathrm{PCO}_{2}$ of $3.52 \mathrm{kPa}$ $(4.7-6.0 \mathrm{kPa}), \mathrm{PO}_{2} 12.95 \mathrm{kPa}(9.3-13.3 \mathrm{kPa})$ and $\mathrm{HCO}_{3} 15.0$ $\mathrm{mmol} / \mathrm{l}(22-26 \mathrm{mmol} / \mathrm{l})$, base excess of -8.5 (-2 to $2 \mathrm{mmol} / \mathrm{l})$. Her anion gap was raised at $18.1 \mathrm{mmol} / \mathrm{l}(8-$ $16 \mathrm{mmol} / \mathrm{l})$ and lactate was normal at $0.6 \mathrm{mmol} / \mathrm{l}(<2 \mathrm{mmol} / \mathrm{l})$. Electrocardiogram (ECG) demonstrated sinus tachycardia with non-specific ST changes in V3-V6. Chest X-ray demonstrated normal heart size and a small area of shadowing in the left middle zone. She was admitted with a provisional diagnosis of viral respiratory infection with pleurisy. She was treated initially with intravenous (iv) fluids.

Urinalysis was strongly positive for ketones $(+++)$. Her full blood count, urea and electrolytes and C-reactive protein were normal. Salicylate levels were negative. A computed tomography pulmonary angiogram (CTPA) showed patchy consolidation of midzones and right upper lobe, and fluid distension within the oesophagus and a pouch of this was isolated by the band, herniating into the hiatus hernia. There was no free fluid or gas in the abdomen and no evidence of pulmonary embolism (Fig 1).

\section{Differential diagnosis}

She had a high anion gap, metabolic acidosis with normal lactate levels. The differential diagnosis of metabolic acidosis is highlighted in Fig 3. Careful consideration of her history (especially not eating and drinking, vomiting), and exclusion of other

A Lulsegged, ${ }^{1}$ consultant physician, endocrinology and diabetes; E Saeed, ${ }^{2}$ foundation year 1, general medicine; E Langford, ${ }^{2}$ consultant physician, cardiology; C Duffield, ${ }^{3}$ foundation year 1 , general surgery; S El-Hasani, ${ }^{3}$ general surgeon; N Pareek, ${ }^{2}$ specialist registrar, general medicine and cardiology

Departments of Endocrinology, ${ }^{1}$ Cardiology, ${ }^{2}$ Surgery, ${ }^{3}$ South London NHS Trust, Princess Royal University, Farnborough Common causes of a high anion gap acidosis (normal lactate, glucose, salicylate levels and no features to suggest other poisoning) led to the suspicion that she had starvation ketoacidosis and aspiration pneumonia.

\section{Initial management}

Investigations were carried out to exclude the different causes of her high anion gap metabolic acidosis including lactate, salicylate levels, blood glucose levels, urine (ideally blood ketone metre readings), calcium oxalate crystals on urine microscopy and/or a high osmolar gap (obtained by comparing the patient's measured serum osmolality against predicted osmolality) may suggest ethylene glycol poisoning especially if clinically
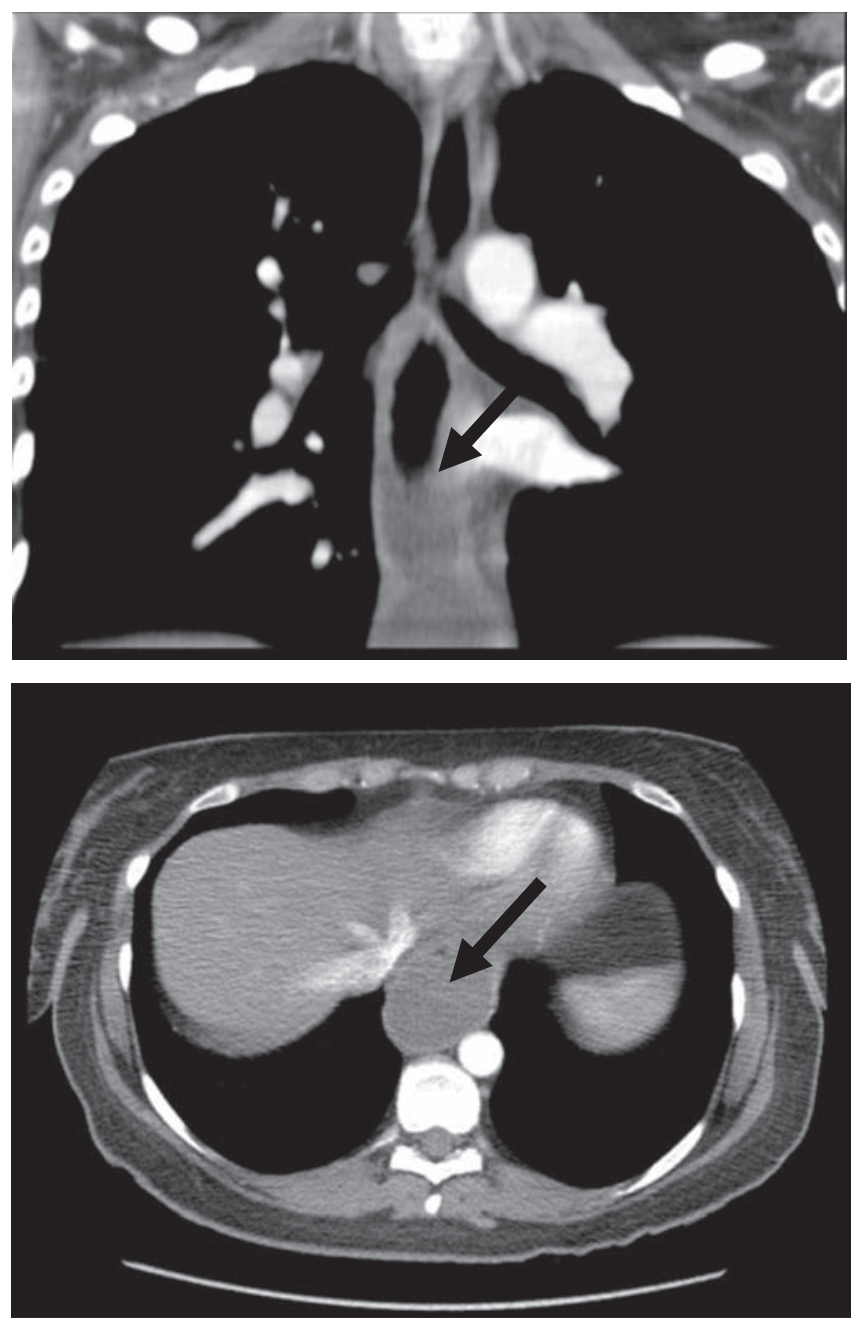

Fig 1. Computed tomography (CT) scan of the thorax showing dilated oesophagus with a pouch isolated by the gastric band. The band had herniated and there was no free fluid or evidence of perforation. 


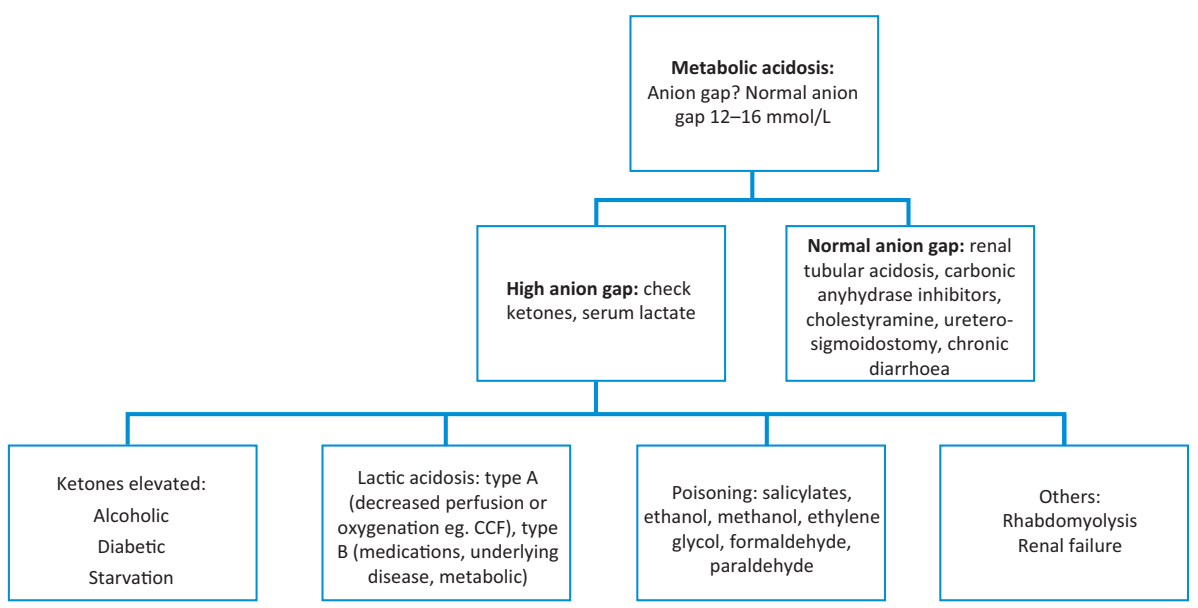

Fig 3. Algorithm for the classification and diagnosis of metabolic acidosis.

corroborating features are present. It is important to appreciate, however, that other substances can cause a high osmolar gap, such as ethanol and methanol, and a normal osmolar gap does not exclude ethylene glycol poisoning. This patient did not have these tests. She was given iv 10\% dextrose and insulin with strict monitoring to maintain euglycaemia.

\section{Case progression}

The following day her gastric band was loosened via the external port and post-procedure contrast studies revealed normal function/no surgical problems. She made a rapid clinical and biochemical recovery and tolerated oral intake within 24 hours. Her blood analyses improved over the next three days (Table 1) and she was discharged to complete a course of oral antibiotics. She has been well at follow-up with no further complications.

\section{Discussion}

Obesity is increasingly prevalent across the world and surgical procedures for this are becoming more common. ${ }^{1}$ Starvation ketoacidosis is a rare cause of metabolic acidosis. There are sev-

Table 1. Arterial and venous blood analysis during admission.

$\begin{array}{lccc}\text { Parameters } & \begin{array}{l}\text { Arterial blood } \\ \text { on admission }\end{array} & \begin{array}{l}\text { Arterial blood } \\ \mathbf{1 2} \text { hours after } \\ \text { admission }\end{array} & \begin{array}{l}\text { Venous } \\ \text { blood on } \\ \text { discharge }\end{array} \\ \mathrm{pH}(7.35-7.45) & 7.32 & 7.289 & 7.436 \\ \mathrm{pCO}_{2}(4.5-6 \mathrm{kPa}) & 3.52 & 2.75 & 5.76 \\ \mathrm{PO}_{2}(11-14 \mathrm{kPa}) & 12.95 & 15.47 & 3.92 \\ \mathrm{HCO}_{3} & 15.0 & 9.7 & 28.4 \\ (22-28 \mathrm{mmol} / \mathrm{L}) & & -14.7 & 3.7 \\ \mathrm{BE}(-2-+2) & -8.5 & 0.57 & 1.17 \\ \text { Lactate } & 0.6 & & 15.3 \\ (<2 \text { mmol/l) } & & 21.3 & \\ \text { Anion gap } & 18.1 & & \\ (10-16 \mathrm{mmol} / \mathrm{l}) & & & \end{array}$

eral case reports of this being associated with gastric banding, ${ }^{2}$ infant intensive care treatment ${ }^{3}$ and pregnancy. ${ }^{4}$ The diagnosis of starvation ketoacidosis is based on the following criteria: history of reduced calorific intake, low plasma glucose concentration, the presence of ketonaemia or ketonuria and a high serum beta-hydroxybutyrate level.

During starvation, the blood glucose concentration is reduced with subsequent decrease in insulin and increased glucagon levels. This leads to depletion of hepatic glycogen stores. Reduced insulin levels promote increased activity of lipase, which, in turn, breaks down triglyceride into free fatty acid chains (FFA). FFA are oxidised in the liver forming ketone bodies. Pathological ketosis causing ketoacidosis is associated with an excess production of ketone bodies over and above what the body can use as an energy source.

The level of ketonaemia in starvation ketoacidosis appears to be relatively mild in comparison to that seen in diabetic or alcoholic ketoacidosis. This is thought to be due to secretion of sufficient, albeit reduced levels of insulin which prevents overwhelming ketoacidosis.

Importantly this case underlines the importance of a careful, methodical approach to interpreting acid base disturbances. This is more so when the aetiology is not initially clear as was the case in this patient. A systematic approach was adopted in

\section{Key learning points}

- A methodical approach, as described in the text can be very helpful in determining the cause of metabolic acidosis and providing prompt medical treatment.

- Blood ketone measurements are more accurate than urinalysis and some patients may not be able to produce urine for analysis when acutely unwell.

- Insulin and dextrose promotes a rapid resolution of the biochemical and acid-base disturbance in patients with starvation ketoacidosis.

- Gastric banding is becoming increasingly common and patients who have them could be at risk of starvation ketoacidosis. 
diagnosing the aetiology of the metabolic acidosis in this patient, as shown in Fig 3.

Management of starvation ketoacidosis is aimed at removing the causative factor and reducing gluconeogenesis. This is achieved by the iv administration of dextrose and insulin, although some have used just dextrose alone. Insulin therapy switches off ketogenesis and may facilitate a more rapid recovery. When used for this purpose it is important to ensure that the patient is given enough dextrose to prevent them becoming hypoglycaemic and careful, regular checks are made to ensure the blood glucose levels are kept in the normal range. There is little benefit in using iv bicarbonate.

\section{References}

1 National Institute for Health and Clinical Excellence. NICE obesity: guidance on prevention, identification, assessment, and management of overweight and obesity in adults and children. London: NICE, 2006.

2 Bruegger D, Rehm M, Da Silva L, Christ F, Finsterer U. Severe metabolic acidosis resulting from a dislocated gastric band. Obes Surg 2004;14:555-8.
3 Toth HL, Greenbaum LA. Severe acidosis caused by starvation and stress. Am J Kidney Dis 2003;42:E16-9.

4 Burbos N, Shiner AM, Morris E. Severe metabolic acidosis as a consequence of acute starvation in pregnancy. Arch Gynecol Obstet 2009;279:399-400.

Address for correspondence: Dr A Lulsegged,

South London NHS Trust, Princess Royal University, Farnborough Common, Orpington BR6 8ND.

Email: alulsegged@nhs.net

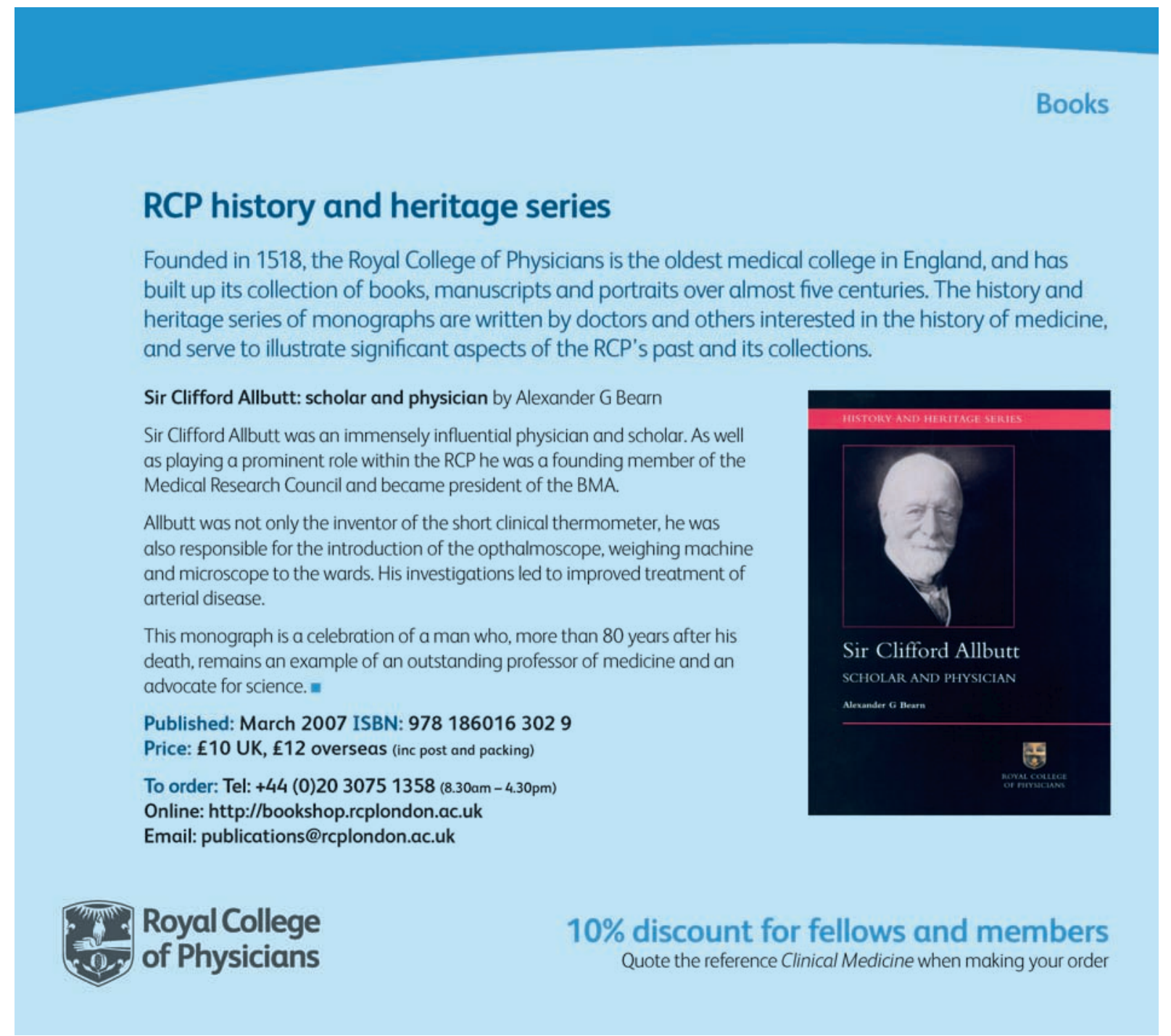

